\title{
A scoping review on the influencing factors and development process of professional identity among nursing students and nurses
}

aimei mao ( $\nabla$ maoaimei@kwnc.edu.mo)

Kiang Wu Nursing College of Macau https://orcid.org/0000-0001-8894-4943

\section{Su-e Lu}

Kiang Wu Hospital

Yan Lin

Kiang Wu Hospital

Miao He

Kiang Wu Hospital

\section{Research article}

Keywords: China, development process, influencing factors, nurses, nursing students, professional identity, scoping review

Posted Date: August 22nd, 2019

DOl: https://doi.org/10.21203/rs.2.13395/v1

License: (a) (i) This work is licensed under a Creative Commons Attribution 4.0 International License. Read Full License

Version of Record: A version of this preprint was published at Journal of Professional Nursing on March 1st, 2021. See the published version at https://doi.org/10.1016/j.profnurs.2020.04.018. 


\section{Abstract}

Background Professional identity is related to individuals' professional commitment. It has been a hot topic in the nursing science because of the common problem of nursing workforce shortage around the world. Professional identity is culturally shaped, but few scholars have systematically examined its developmental characteristics in a specific culture. The aim of the scoping review is to get comprehensive knowledge on the influencing factors and development process of the professional identity among nursing students and nurses in mainland China

Methods A scoping review was conducted. The most common Chinese databases, China National Knowledge Infrastructure and Wanfang Data, were searched for publications in Chinese. The EBSCOhost and ProQuest dissertation and thesis global were searched for publications in English. After screening the title and abstract of the articles in the first round and the full-text in the second round, 53 articles were included for analysis.

Results The influencing factors to professional identity development in nursing could be grouped into three dimensions: personal factors at micro dimension, familier factors and institutional factors at medium dimension, and social factors at macro dimension. The social factors tended to negatively affect professional identity while the factors at the other two dimensions exerted influence in different directions. A framework was established based on professional identity levels in different career stages of nurses to depict the continuum and dynamic nature of development process.

Conclusions Development of professional identity in nursing is a dynamic process shaped by multidimensional factors. The biggest obstacles to the development lie in the social factors. Changes in policy should be made to reverse the nursing profession stereotype of being a passive role to medicine. As some obstacles and challenges faced by nursing are shared by nurses around the world, international cooperation is needed to address the common obstacles and challenges. Keywords: China; development process; influencing factors; nurses; nursing students; professional identity; scoping review

\section{Background}

Formation of professional identity (PI) is the result of professional socialisation, a process in which the individuals learn and internalise profession related knowledge, skills, norms, and values $(1,2)$. It is estimated that by 2030 there will be shortage of 18 million healthcare professionals, among whom 50\% being the nurses (3).Nursing leaders worldwide have called for governments to pay immediate attention to the issue of retention, given the bitter fact that the current nurses are leaving their profession at a pace faster than ever (3). Studies have confirmed the correlation between PI and retention of nursing workforce. Higher levels of PI, sometimes called positive PI, are associated with higher commitment to profession (4-6). China hosts 3.8 million of registered nurses, the largest number in the world. However, 
compared with its huge population of 1.4 billion, China is among the countries with the lowest nurse/population ratio, with 2.74 nurses per thousand of population (7). In addition to increasing recruitment, cultivating positive PI among prospect and current nurses is essential in China to prevent turnover of nursing workforce.

There are research studies in China on the development of PI among nursing students and clinical nurses. For example, the study by Ren et al. (8) on 423 nurses in three large hospitals in a city of Southwestern China found that the PI development of the nurses was influenced by their work positions, marital states and nightshifts. He et al. (9) studied 582 nursing students in the final year of the clinical placement stage and found that a variety of factors contributed and affected the PI of the students, such as age, program type, mothers' education, motivations to choose nursing, participation in students' associations, academic performance, teachers' teaching manners, salary levels of local nurses, perceived social status of nurses, etc. While these studies have enriched knowledge on the professional socialization of nurses they usually examined different influencing factors with non-representative participants. Furthermore, some studies in China have measured the PI levels with nurses in different stages of their career life. It is worthwhile to conduct review studies to provide a whole picture of the experiences of nursing students and nurses in constructing their PI.

This review will add cultural diversity of PI construction. It will answer two questions: 1) Which factors contribute to the construction of PI among Chinese nursing students and nurses? 2) What is the PI development process like? There are significant differences in respect to nursing education and practice in different regions of Greater China, which includes Hong Kong, Taiwan, Macau, and mainland China. Here we focus on the nursing students and nurses in mainland China, where the number of nurses largely outweighs the combined number of the nurses in the other three regions. "Mainland China" is usually simply called "China"; so these two words are used interchangeably in this article.

\section{Methods}

A scoping review was conducted on the published literature on nursing PI development in China. The reviewing framework proposed by Arksey et al. (10) guided the reviewing process. 
Stage 1: Identifying the review questions.

As aforementioned, our review aims to gain knowledge on the general experiences of Chinese nursing students and nurses in developing their PI. Two research questions were defined: 1) Which factors contribute to the construction of PI among nursing students and nurses? 2) What is the PI development process like?

Stage 2: Searching for relevant literature

We searched the most popular Chinese databases, China National Knowledge Infrastructure and Wanfang Data, for published literatures inside China. English databases EBSCOhost (including CINAHL Complete, Medline, PsycINFO etc. 28 databases) and ProQuest Dissertation and Thesis Global were searched for the literatures published outside China. There was no publication time limitation. The keywords < "professional socialization" or "professional identity" or "professional development" > combined with < "nurses" or "nursing students" or "nursing"> were used in literature search. Chinese synonyms were used to maximise the inclusion. There were 18 different combinations, resulting in 1605 articles identified. "Chinese or China" > were added to search the relevant publications in English databases and 341 articles were identified.

Stage 3: Screening the articles

After excluding the duplicates, we developed inclusion and exclusion criteria for the screening process. The inclusion criteria were: 1) studies on influencing factors of nursing PI development; 2) Studies on development process of nursing PI. The exclusion criteria were: 1) Studies only on PI of special groups, such as male nurses; 2) Studies only on nursing students of non-bachelorette degree, because bachelorette degree program is becoming the most common program of nursing education in China. Associate diploma program is shrinking and the number of postgraduate program attendees is still very small; 3) Studies of poor quality. Scholars have argued that lack of quality assessment of the included studies may jeopardize the legitimacy of the review findings (11). Practically, we encountered a large number of studies identified and decided appraising the included studies as a necessary process.

We applied a two-round of screening process. The first round of screening was based on title and abstract of the included articles and the second round was based on the full-text of the 
articles left after the first round of screening. Three members of our research team did the screening independently. The four members of the team met again and got consensus on the final screening results.

Selection of the included articles from English databases was conducted after the screening of Chinese studies. Screening articles in English was challenging for some of the team members because of their poorer English. Given that the selecting standards had become stable after the articles from the Chinese databases had been screened, we decided to have one of the team members to do the English articles screening.

After the two rounds of screening of all articles from both Chinese databases and English databases, there were 47 articles included. A manual search was then undertaken to identify more eligible articles. Seven articles were added to the included list. Figure 1 is the flowchart of searching and screening of the relevant literatures described above.

This process involves sorting materials according to research questions. We utilized Nvivo, a qualitative research analytic software to facilitate the sorting process. We picked up the essential information from the included articles, such as type of the artilces, background of the authors, publishing time, the purpose of the study, research design, and research findings, etc.

Stage 5: Synthesize the findings.

Again this is a similar process to data analysis in qualitative research. We read through the full-text of the articles and used inductive and deductive techniques in data analysis. There are two themes related to the research questions, "influencing factors to PI development" and "development process of nursing PI".

\section{Results}

\section{The overview of the included articles}

There were two literature reviews $(12,13)$ and the other 51 articles were non-experimental descriptive studies. Forty-two (80\%) of the 53 articles were survey studies conducted either in 
nursing schools or/and in hospitals, with the number of participants ranging from 200-1983. Only seven surveys applied random sampling. Studies conducted with clinical nurses all happened in large- or middle- sized hospitals in metropolitans or the regional capital cities. Despite no publication time imposed, the articles were published from 2006-2018, with more articles published in the last four years. The overview of the articles was shown in Table 1.

Table1 Basic information of the included studies $(\mathrm{N}=53$ [ 
Study design

Survey

Random sampling

$7 \quad 13.2$

Convenience sampling

$35 \quad 66.0$

Qualitative research

Personal interview

$3 \quad 5.7$

Focus group

11.9

Mixed design

Survey + personal interview

$3 \quad 5.7$

Survey +literature review

11.9

Survey + case study (general) literature review

Academic background of the first author

Nursing

Nursing schools (including adult students in masters or doctorate programs)

Hospitals

Management

Participants of the studies

Nursing students

Clinical nurses

$14 \quad 26.4$

11.9

$28 \quad 52.8$

Tertiary hospitals*

$12 \quad 22.6$

Other hospitals in metropolitan or capital cities

Mixed participants (nursing students and clinical nurses)

Publication sources

Academic journals

Nursing journals

Other journal

University /college journals

Master's degree thesis

Other medical journals

Publication time $\square 2006-2018 \square$

2014 and onwards

2009-2013

2008 and before
$10 \quad 18.9$

$\begin{array}{ll}3 & 5.7\end{array}$

$29 \quad 54.7$

$5+113^{9}$

$31 \quad 58.5$

$\begin{array}{ll}17 & 32.1\end{array}$

$5 \quad 9.4$
$13 \quad 24.5$

*There are different hospital rankings in China and the biggest and best are called "tertiary hospital".

Influencing factors to PI development 
All the included survey studies applied at least one instrument to explore PI related elements. We focused on the studies with the instruments specifically identifying PI levels and influencing factors based on our research questions. Researchers in the included studies applied nine PI instruments targeting nursing students and clinical nurses, among which seven were developed by the nursing scholars in China with the other two being translated and modified from the instruments outside China. Items in the PI instruments were different but generally embraced such elements as self-identity, perceived professional autonomy, perceived professional competency, feelings to profession, professional expectations, etc. The number of the dimensions with the instruments were 6-7, with the total number of items ranging from 17-44. Likert rating scale was used to measure the PI levels of the student and/or nurse participants. The higher score the participants got, the higher PI level they had.

While the individual survey studies explored only some of influencing factors to nursing PI, combination of the findings from the studies can provide a whole map. The factors can be grouped into three levels of dimensions: micro, medium and macro. The different dimensions of the factors are interplayed, exerting a combining force to shape the construction of PI (Figure 2).

Personal factors affecting nursing PI development

Personal factors were mentioned by all the included articles. The most frequently examined factors were basic information, such as age, gender, motivations to join nursing, year of stud or year of work, and type of program joining in. Gender differences in PI development were reported by those studies with mixed gender participants. Nursing was traditionally regarded as the care work carried out by females and male nursing students and male nurses had lower level of PI. Motivations to join nursing was another definite contributor to PI, with those who were fond of nursing and willingly joined the profession showing higher PI in both nursing students and clinical nurses.

One of the common findings was the declined PI levels among the students with their increased length of academic study. The fresh students had the highest level while the senior students had the lowest. This was because the fresh students held ideal image on nursing and nurses while the seniors experienced the gap between the ideals and realities. Studies with participants from different programs got the finding that the students in lower degree of 
programs had higher level of PI. Those students in higher degree programs tended to hold higher expectations of themselves and might experience the gap between the expectations and the reality. One positive factor to PI reported unanimously by the studies was holding a position. The nursing students who held a leader's position or the nurses who held a management or expert position had higher PI, because holding a position would enhance the overall self-confidence of the individuals.

The studies examined marital status and age but reported mixed results. Two studies examined the influence of being the single child of the family to nursing PI $(14,15)$. China relaxed it's One-Child Policy in 2015 and a large proportion of the current youths and adolescents are the singletons as the result of 35 years of implementation of the policy. The studies found that the singletons suffered lower level of PI because of the tough nature of nursing job. Nurses usually worked at busy and stressful scenarios. This working environment was the sharp contrast to the cosiness enjoyed at home by the singletons.

\section{Familier factors affecting nursing PI development}

All the included studies sent a strong message that family support was significantly associated with higher PI. Specially, the nursing students who had family members working in health care showed higher PI (16-18). There are controversial findings over whether the family economic conditions have impacts on PI of nurses The comfort in the affluent household would be contrary to the stressful working conditions of nursing, resulting in nurses' dissatisfaction with their job, which would in turn result in lower PI among nurses from richer families (19). On the other hand, nurses from richer family were found to be more committed to nursing job because they had chosen nursing out of fondness(17). Several studies found that nightshift was an influencing factor to lower PI levels (20-22). Qualitative studies provided nurses' own explanations that working on nightshift somehow limited their time to take care of family $(20,23)$.

Institutional factors affecting nursing PI development

Studies exploring the institutional factors had no consensus findings. Some studies found higher PI among those nurses with higher salaries $(6,16,20,24,25)$. This happened among the nurses in the same health institution because salaries were not comparable among nurses 
in different institutions. Being awarded more than colleagues meant higher professional recognition, which could be transferred into higher self-identity. Despite the good feelings with some nurses with higher salaries, nurses as a whole were unsatisfied with reimbursement in their institutions. The nurse participants in the included qualitative studies complained of much lower salaries with nurses than other professionals in the same working unit $(20,26)$.

Several studies found the impacts of nature of hospitals and employment on nursing PI $(6,16$, $27,28)$. With the market economy expansion in China in the past forty decades, there are increased private hospitals. However, public health institutions generally enjoy more resources than private ones. The nurses in the public hospitals work on two kinds of employment: formal employment and informal employment. The former is called bianzhi (ㅁ) while the latter is called contract-based. The employees on bianzhi enjoy higher salaries, more secure position, and more promotion opportunities. The limited number of studies revealed that the nurses in public hospitals enjoyed higher PI than those in private hospitals and that the nurses on bianzhi enjoyed higher PI than those on contract-based $(22,27)$.

The hospital management manner was another influencing factor to nursing PI. As it was difficult to quantify management manner this factor was implicitly embedded in the participants' accounts in the included qualitative studies $(14,20,26)$. Nurses were unhappy with the nursing management manner. There were no boundaries of responsibilities for nurses and they ended up to doing everything left by other professionals. Nurses' voice was dumb or weak in decision making in the multidisciplinary medical or management team and they were treated unfairly if something wrong happened. It would be the nurses who were to be blamed, not the physicians, if the latter made an order mistake, because the nurses did not find out the mistake.

\section{Social factors affecting nursing PI development}

None of the included quantitative studies explicitly explored social factors. However, the accounts of the participants in the included qualitative studies clearly showed the impact of social factors, which negatively affected the nursing PI development $(14,20,26,29)$. Senior nursing students and clinical nurses mentioned that nursing was not regarded as a decent job in China. Nurses were not respected by patients and other medical professionals. When 
patients discharged after they were cured they would thank the physicians with no mention of nurses' contributions. Nursing currently still subordinated to medicine and the public regarded nurses as physicians' assistants, doing trifles under the physicians' orders.

\section{Development process of nursing PI}

Researchers have suggested that professional socialisation is a subtle process, unnoticeable to the participants $(1,2)$. In-depth analysis of the included studies with participants of various stages of professional development provided clues of development process of PI in nursing.

The review found the highest level of PI among the fresh students (29-32). This indicates that conceptions on nursing and nurses prior to formal nursing education shaped the PI of those beginners. However, an interesting and common finding across the studies was the declining PI levels as the students advanced professional studying. The increased professional knowledge and skills seemed not to elevate nursing students' confidence in proving nursing care when they learned more about the practicing complexity.

The dynamic nature of PI development was also detected with clinical nurses. Despite contradictory findings, more studies supported higher PI among the nurses in the first five years of practices $(16,22,33-36)$. Newly graduated nurses might experience dramatic role changes in the first years. They had to rotate in different wards, learning specific nursing skills and routines in different wards. It is supposed that after the first one or two years of transition time, the nurses came out more confident in their professional proficiency and better adapting to the environmental complexity. Also, as the new professionals they held expectations for career development. All these would have contributed to the higher PI among the new nurses.

After nurses had worked for several years they were more familiar with the practice routines and more competent in nursing care. However, their passion to the profession begun to fade. Also, they had to balance their professional and family roles, given that, at this time, the nurses usually had to take care their children. They were likely to become burnouts under the dual burden of work and family.

Senior nurses who had worked more than 20 years might have been promoted to management or expert positions. Those who did not get promoted might feel challenging to 
continue frontline nursing and had to move to other positions less physically demanding. One study exclusively explored 474 nurses who had worked for 20-35 years (37). It was found that all the nurses had middle or high-level professional qualifications and $32.1 \%$ of them were in management position. None of them intended to leave nursing. At the same time they felt physically unsuitable for the frontline nightshifts and were unhappy with the same professional role as the junior nurses. Some of them had no expectations of career development and looked forward retirement.

The included studies examined the nurses' levels of PI using different instruments with different range of scores and it is impossible to compare the PI levels of nursing students and nurses in different studies. The researchers usually compared the participants' PI levels with the medium score. For example, in a Likert rate of 1-5, 1-2 was regarded as very low score, with 2-3 as low, 3-4 as medium and 4-5 as high. The participants' scores generally fell on 3-4 score range. The scores fluctuated around the medium level. The dynamic nature of PI development of nurses at different stages of career life can be demonstrated in figure 3. Figure 3: The PI developmental levels in different stages of career life of Chinese nurses

\section{Discussion}

Although there are numerous studies outside China exploring the PI development among nursing students and frontline nurses, very few studies have done the similar studies as in China to examine influencing factors in different dimensions and to measure the PI levels. Our review has made further contribution by synthesizing the existing studies and provide a whole picture of the experiences of PI development among Chinese nursing students and nurses. The review found that, despite ups and downs of PI levels in the different stages of professional lifetime, the PI levels fluctuate around the medium levels of PI scores in different measurement tools. This indicated the overall low passion to nursing among nursing students and clinical nurses in China. A study compared the PI levels of the nursing students from Hong Kong and mainland China and found that students from Hong Kong enjoyed much higher PI levels than their mainland counterparts (38). The turnover rate among Chinese nurses is reported at $5.8 \%$ (7). Compared with $10-40 \%$ of turnover rates in other countries (39-41), China seems enjoying a relatively higher rate of retention. It is not enjoyable, 
however, that the higher retention is accompanied with nurses' low level of passion to nursing profession.

A review by Hoeve et al. (42) found low self-esteem and low PI as a common sign among the nurses all over the world. They concluded that the low social status of nursing profession had contributed to the low PI among nurses. Our review revealed that the social factors exerted an overall negative influencing force to PI development. Scholars have suggested that PI is shaped by the perceived image of others on the profession (43). Traditional Chinese medicine attached important role to nursing in medical care but regarded nursing as a family-based care, carried out by female family members without professional knowledge and skills. This has helped to shape nursing as subordinate to medical treatment, which is traditionally performed by males. Nursing gained the status of a profession in China at much later time than many other countries. Our review shows that perception of nursing as a profession has not yet accepted by the public.

The development process framework of nursing PI established by our review tries to demonstrate the professional socialization in a clear and vivid way. The framework supports Miller's theory that PI is formed before the prospect professionals enter formal professional education and last their whole career lifetime (1). However, Miller's theory mainly focuses on formal educational time and does not mention PI developmental characteristics of postgraduation. Our framework has enriched Miller's theory by depicting the developmental characteristics of PI in more details, particularly at the post-graduation time period. Okura et al. (44) also suggested a five-stage process about the PI development among public health nurses, similar to Benner (45) suggested five stages of PI development for any profession: novice, advanced beginner, competent, proficient, and expert. The five-stage PI development theories focus on the post-graduation time period and are based on the professional proficiency. This is different from our framework, beginning from pre-education time throughout the whole career life of nurses. Our review does not find the increased PI associated with the advancement of professional proficiency, indicating that PI is a set of traits more than the component of professional proficiency.

\section{Implications for nursing practice}


Our searching results revealed the inappropriately distributed research resources on nursing PI in China. The majority of the included studies with clinical nurses were conducted at the large hospitals in metropolitans and regional capital cities. Future research resources should be allocated to the under investigated small and middle hospitals so that a fuller picture on nursing PI can be captured. Further, more rigorously designed studies should be carried out. For example, studies with sufficient participants through random sampling from different regions. While qualitative research studies outside China on nursing PI is flourishing (42), this type of research is underdeveloped in China. It can be designed standing along or as complimentary to quantitative research.

The finding that PI decreases with the academic advancement of the nursing students should be taken into account in nursing education. The nursing schools should provide accurate information about both advantages and disadvantages of nursing jobs during recruitment activities $(46,47)$. Adequate knowledge about nursing will help the high school graduates to make informed decisions on joining nursing at their own will. While "reality shock" is the common phenomenon among the students of different professions when they enter the practice world from classroom $(46,48)$, early exposure to the reality seems reasonable to reduce the shock. While clinical study consists of an important part in nursing curricula, the nursing schools in China have the clinical arrangement almost solely in the last year. Senior students may experience tremendous stress after they suddenly step into the reality, complicating their PI construction process. Chinese nursing scholars have suggested increased early exposure to clinical world for junior students (49). Decreased PI among nursing students also attributed to different teachings offered by school teachers and clinical teachers, with the former tending to teach ideal knowledge and the latter providing information on actual doings $(50,51)$. The two groups should cooperate in teaching so as to provide nursing students coherent information.

Nurses as a whole have been marginalized in the hierarchical power system of the health institutions $(52,53)$. However, nursing managers, who represent nurses' interest, should raise their voice. They can provide evidence for need of recruitment of nursing workforce and for improvement of nurses' benefits. There have been discussions in China over equal pay in equal position, regardless of bianzhi or contract-based employment (54-56), and nursing 
managers should actively involve in such discussions. Older nurses have experiences in nursing care but in the heavy-burdened clinical environment their professional proficiency has been overlooked while their physical frailness is amplified. Nursing managers, together with nursing researchers, should look for ways to make use of expertise of the older nurses as nursing is becoming more specialised.

Our review found that low social status of nursing was the most important obstacle to positive PI development among nurses. Scholars have called for nurses to be politically engaging to change or reverse the stereotype of nurses as subordinate to physicians $(42,57)$. Nurses can initiate or take part in the activities to enhance nursing's social status, including disseminating nursing practice to the public in social media platforms, joining public or professional associations to raise their voice, exploring new practicing territories, doing research to advance the profession, etc. As many of the formidable problems faced by nursing, such as short of workforce, lack of professional role boundary, lack of professional autonomy, etc., have been experienced and are still been experiencing by the nurses around the world, cooperation of nurses from different countries and region are needed to seek the solutions $(42,57)$.

\section{Limitation of the study}

As scoping review is relevantly new in the nursing academic arena in mainland China, our review provided a methodological example for future scope reviews on nursing science. However, there are two limitations with the review: 1) Screening of the articles from English databases was conducted by only one member of the research team. Although the screening standards had become stable after screening the articles from Chinese databases had finished, the screening results of the articles from English databases might have been misjudged without peer verifications; 2) The review only selected those articles which explicitly explored the influencing factors to PI in nursing. Other studies which did not overtly examined the PI but explored the factors related to some traits of PI might have been missed out, for example, the studies on the contributors to nurses' burn out /fatigue or the surveys on contributors to nurses' intentions to leave job.

\section{Conclusions}


Nursing researchers in mainland China have made efforts exploring the characteristics of PI development among nursing students and clinical nurses. The current studies are mostly nonexperimental deigned descriptive ones. More rigorously designed studies with representative participants are needed to provide more accurate and generalizable findings. Our review revealed the development of PI of nurses as a dynamic process shaped by multidimensional factors. A relatively low level of PI among Chinese nurses was found and the level did not increase with nurses' enhanced professional proficiency. The review proposed a development framework of PI among Chinese nurses, from which future researchers could begin or enrich their own analysis on PI development and contributing factors. Having identified the facilitators and obstacles to PI development the review provided suggestions to construct positive PI among nursing students and nurses. social factors are the biggest obstacle to nursing PI and nurses should be active to change the profession stereotype of being a passive role to medicine. The identified influencing factors and development process of PI among nurses were not new to nurses outside China, but with characteristics under the cultural and social contexts of China. As some obstacles and challenges faced by nursing are shared by nurses around the world, international cooperation is needed to address the common obstacles and challenges.

\section{Declarations}

\section{Ethics approval and consent to participate}

Not applicable

Consent for publication

Not applicable

\section{Availability of data and material}

The datasets used and/or analysed during the current study are available from the corresponding author on reasonable request.

\section{Competing interests}

The authors declare that they have no competing interests

\section{Funding}


None

\section{Authors' contributions}

AM designed the study. AM, SL, YL and MH identified and screened the literatures. They all analyzed the data. AM was a major contributor in writing the manuscript. All authors read and approved the final manuscript

\section{Acknowledgements}

The authors thank Drs. Mio Leng Au and Yao Chen Chung from Kiang Wu Nursing College of Macau for their revision suggestions for the manuscript.

\section{References}

1. Miller SE. A Conceptual Framework for the Professional Socialization of Social Workers. Journal of Human Behavior in the Social Environment. 2010;20(7):924-38.

2. Van IK. The Impact of a Nursing Program on the Formation of Nursing Students' Professional Identity [Ph.D dissertation]: The Chinese University of Hong Kong (Hong Kong); 2014.

3. International Council of Nurses. ICN International Workforce Forum calls for urgent action from governments to address global nursing shortage 2019, https://www.icn.ch/news/icn-international-workforce-forum-calls-urgent-actiongovernments-address-global-nursing July 42019

4. Sabanciogullari S, Dogan S. Relationship between job satisfaction, professional identity and intention to leave the profession among nurses in Turkey. Journal of Nursing Management. 2015;23(8):1076-85.

5. Lu H, Zhao Y, While A. Job satisfaction among hospital nurses: A literature review. International Journal of Nursing Studies. 2019;94:21-31. 
6. Hu ZH, Ba ZM, She YF. An investigation of professional identity among the clinical nurses in Chengdu. Chinese Health Service Management. 2014(04):311-2,9.

7. People's Daily. Nursing workforce in millions of shortage, low salaries causing loss 2018 http://politics.people.com.cn/n1/2016/0520/c1001-28365329.html March 242019

8. Ren J, Xie Y, Cui WL, Luo S, Yang WQ, Zhang QL. Career identity and its influencing factors among nurses in third-grade class-A hospitals of Panzhihua City. Occupation and Health. 2016;32(18):2537-9.

9. He LY, Dong CM, Xiao X. Professional identity of nursing students in Hunan province and its influencing factors. Chinese Journal of Nursing Education. 2017;14(04):314-8.

10. Arksey H, O’Malley L, Baldwin S, Harris J. Services to Support Carers of People with Mental Health Problems: Literature Review Report. London: National Co-ordinating Centre for NHS Service Delivery and Organisation; 2002.

11. Levac D, Colquhoun H, O'Brien KK. Scoping studies: advancing the methodology. Implementation science : IS. 2010;5:69.

12. Chen HZ, Zhang CY, Ban JJ, Zhang P. Research status quo and related factors of nurses professional identity. Modern Clinical Nursing. 2015;14(7):77-80.

13. Hu YY, Qiu YQ, Hu ZP, Du SP, Shen YY, Yu XL, et al. A review on the professional identity of the undergraduate nursing students. Nursing Journal of Chinese People's Liberation Army. 2009;26(24):47-8,56.

14. Li YL, Zuo HX, Chen QS. Quantitative and qualitative research on occupation identity of new nursing undergraduates. Chinese Nursing Research. 2012;26(23):2133-5.

15. Liu YL, Li QW, Chen N, Feng HD. The professional self-identity and influencing factors among baccalaureate nursing students. Journal of Shenyang Medical College. 2013;15(3):168-9.

16. Gao.H. An exploration on the professional identity levels and influencing factors among 921 clinical nurses from 20 hospitals in Shanghai. Journal of Nursing. 2013;20(16):27-30.

17. Tian YX, Zhu JH, Zhang L. Professional identity of nursing students in Ningxia province and its influencing factors. Journal of Nursing Science. 2012;27(1):69-70. 
18. Fan ZH, Wang XY, Hou RP. A survey on the professional identity status and influencing factors among baccalaureate nursing students in Henan Province. Health Vocational Education. 2018;36(19):126-7.

19. Tang XL, Wang YR, Li XJ, Hu ZY. A study on the professional identity status and influencing factors among the newly enrolled students in four nursing schools in Inner Mongolia. Journal of Nursing. 2013(14):22-4.

20. Cai XC. Study on the current condition and influence factors of nurse professional identity [Thesis]: Shan Xi Medical University 2012.

21. Liu CX, He CY. The level and influence factors of professional identity among junior Nurses. Chinese Journal of Nursing Education. 2015;12(11):813-6.

22. Xu BF, Cui LJ, Wang YL. Status Survey Analysis on the Sense of Professional Identity of Recruiting Nurses. Chinese Medical Record. 2013;14(6):43-5.

23. Xia Y. A study on professional identity of nursing students of Shanghai Jiao Tong University [Master's thesis]: Shanghai Jiao Tong University; 2010.

24. Luo ZN, Li SZ. The professional identity and the influencing factors among the nurses in the hospitals in Guangzhou Psychological Doctor. 2016;22(13):233-5.

25. Jiang YH, Wang GP. Research of the Professional Identity of Nurses in Urban Secondary Comprehensive Private Hospitals. Journal of Shenyang Medical College. 2018;20(3):22932.

26. Deng LC, Duan Y. The status of professional identity among baccalaureate nursing students and strategies Journal of Ezhou University. 2015(10):110-2.

27. Zhang P, Sun XM, Chen HZ, Pu XJ, Pei YJ. Exploration of professional identity and the influencing factors among the nurses in different hospitals in Gansu Province Health Vocational Education. 2016;34(12):85-7.

28. Zhang CY, Lu GF, Chen RR, Liu.L., Xu XN. Investigation and analysis of the clinical nurse status of professional cognition in three top hospitals. Journal of Lanzhou University (Medical Sciences). 2010;36(02):85-8.

29. Miheriayi A, Parida M. Status quo of professional identity of nursing students at different levels and different nationalities in universities in Xinjiang. Chinese Nursing Research. 
2017;31(05):605-7.

30. Wang SD, Gu LL, Huang HX, Liang YY, Wang HP. Investigation on professional identity of undergraduate nursing students from Inner Mongolia region. Journal of Nursing Science. 2016;31(24):82-3.

31. Du L, Lu GB. The level and the influence factors of professional identity among undergraduate nursing students. Chinese Journal of Nursing Education. 2016;13(12):8947.

32. Zhang $\mathrm{X}, \mathrm{He} \mathrm{HR}$, Wang YQ, Pu CX. A comparison of professional identity levels among the nursing students of different programs Health Vocational Education. 2015;33(2):127-9.

33. Zhao H, Zhang CY, Lu TT, Shi Y, Zheng JH, Ding Y, et al. Survey on Career Identity of Nurses in 6 Class $\square$ Grade $\square$ Hospitals Journal of Nursing. 2011;18(7):27-30.

34. Wu.R., Lin M. Investigation on the current situation of career identity of nurses in large Third-grade class-A hospitals. International Journal of Nursing Sciences. 2017;36(8):10304.

35. Li Y, Shan QY, Cai ZX, An H, Wang XQ, Ye Y. Investigation analysis on professional identity and strategies for nurses in Wenzhou. Nursing and Rehabilitation Journal. 2010;9(3):199-201.

36. Fang Y, H., Chen XQ, Jia XE. Investigation and analysis on present situation of nurses' professional identity. Nursing and Rehabilitation Journal. 2014;13(04):320-2,5.

37. Zhang ZX, Shang LP. Analysis of pofessional development of senior nurses and its influencing factors. Chinese Nursing Research. 2015;29(11C):4206-9.

38. Bao JM, Jia YH, Yang F, Han HY, Zhong SQ. Comparison on professional sense of approval between undergraduate nursing students from Hongkong and Hangzhou and enlightenment. Nursing and Rehabilitation Journal. 2014;13(3):212-5.

39. Dewanto A, Wardhani V. Nurse turnover and perceived causes and consequences: a preliminary study at private hospitals in Indonesia. BMC Nursing. 2018;17(2):52.

40. The Sentinel Watch. Nursing Turnover and Retention Strategies 2018, https://www.americansentinel.edu/blog/2018/01/23/nursing-turnover-and-retentionstrategies/ May 222019 
41. Hayes LJ, O’Brien-Pallas L, Duffield C, Shamian J, Buchan J, Hughes F, et al. Nurse turnover: A literature review - An update. International Journal of Nursing Studies. 2012;49(7):887-905.

42. Hoeve Yt, Jansen G, Roodbol P. The nursing profession: public image, self-concept and professional identity. A discussion paper. Journal of Advanced Nursing. 2014;70(2):295309.

43. Tajfel H, Turner JC, editors. The social identity theory of intergroup behavior. New York: Psychology Press; 2004.

44. Okura M, Uza M, Izumi H, Ohno M, Arai H, Saeki K. Factors that affect the process of professional identity formation in public health nurses. Open Journal of Nursing. 2013;3:815.

45. Benner PE. From Novice to Expert: Excellence and Power in Clinical Nursing Practice. commemorative ed ed. Prentice Hall: Upper Saddle Rive; 2000.

46. Jahromi ZB, Jahanbin I, Sharif F, Ghodsbin F, Keshavarzi S. Investigation of the professional self-concept of nursing students in shiraz, iran. Central European Journal of Nursing and Midwifery. 2014;5(3):112-6.

47. Lou SH, Lin XY, Li K. Survey on Professional Identity of Full-time Nursing Undergraduates in Guangzhou. China Higher Medical Education. 2015(10):18-9.

48. Guo B, Zhao L, Gao Y, Peng X, Zhu Y. The status of professional identity and professional self-efficacy of nursing students in China and how the medical documentaries affect them: A quasi-randomized controlled trial. International Journal of Nursing Sciences. 2017;4(2):152-7.

49. Deng FF. Comparison of nursing education among different countries. Chinese Nursing Research. 2015;2(4):96-8.

50. Hao Y-F, Niu H-J, Li L-P, Yue S-J, Liu X-H. Measurement of professional identity in Chinese nursing students. International Journal of Nursing Sciences. 2014;1(2):137-44.

51. Miller SE. Professional Socialization: A Bridge Between the Explicit and Implicit Curricula. Journal of Social Work Education. 2013;49(3):368-86. 
52. Mishra S. Respect for nursing professional: silence must be heard. Indian Heart Journal. 2015;67(5):413-5.

53. ten Hoeve Y, Jansen G, Roodbol P. The nursing profession: public image, self-concept and professional identity. A discussion paper. Journal of Advanced Nursing. 2014;70(2):295309.

54. Yang YH, Ma XJ, Wu Q. Practice and Recommendation of Public Hospital Personnel Filing System Reform Chinese Hospital Management. 2017;37(8):1-3.

55. Zhou Y. Strategies on human resource management in the public sectors-an example from Beijing Human Resource Management. 2016(1):25-6.

56. Zhao J. The current study of nursing human resources of second-class or above comprehensive hospitals in Shanxi [Master thesis ]: Shanxi Medical University; 2015.

57. Premji SS, Hatfield J. Call to Action for Nurses/Nursing. Biomedical Research International. 2016;2016:3127543-.

\section{Figures}




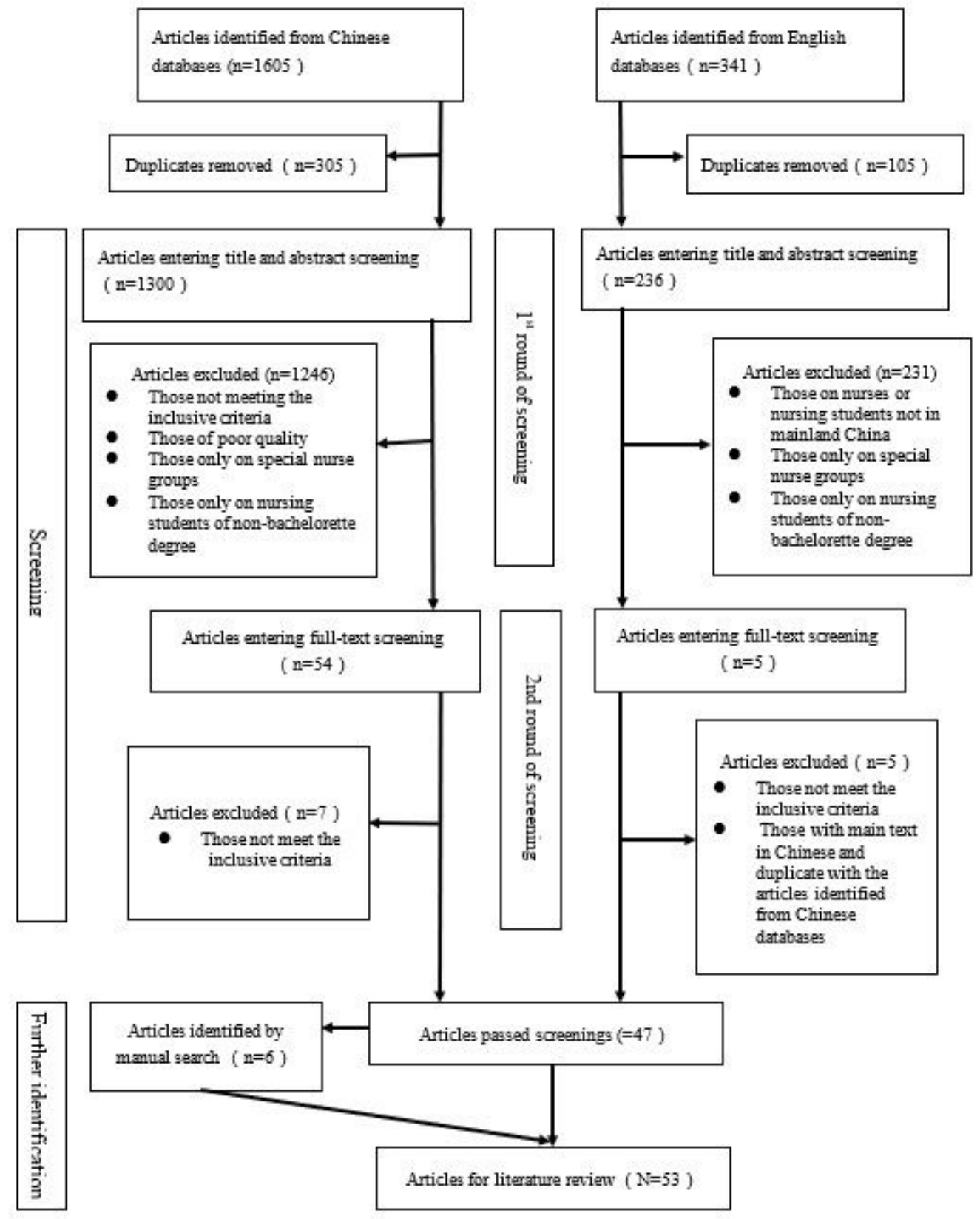

Figurel: Searching and screening procechures for the relev ant publications

\section{Figure 1}

Searching and screening procedures for the relevant publications 


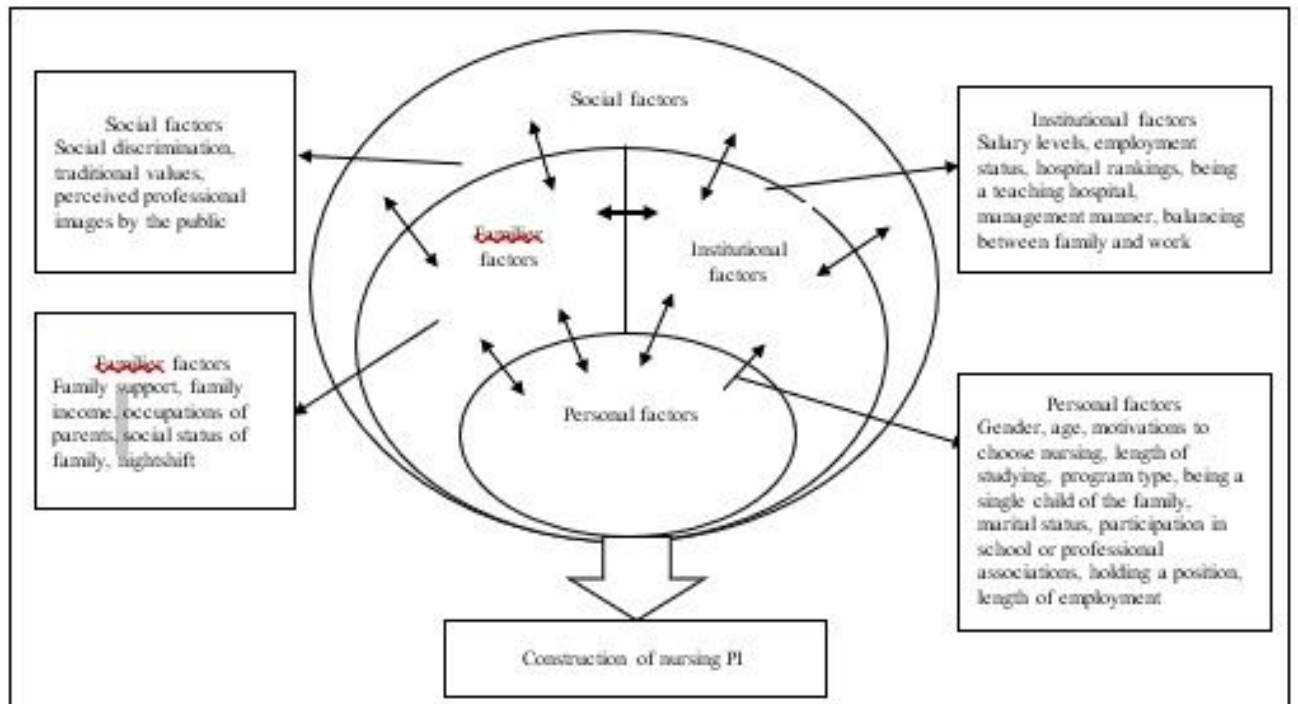

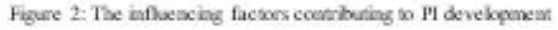

\section{Figure 2}

The influencing factors contributing to PI development

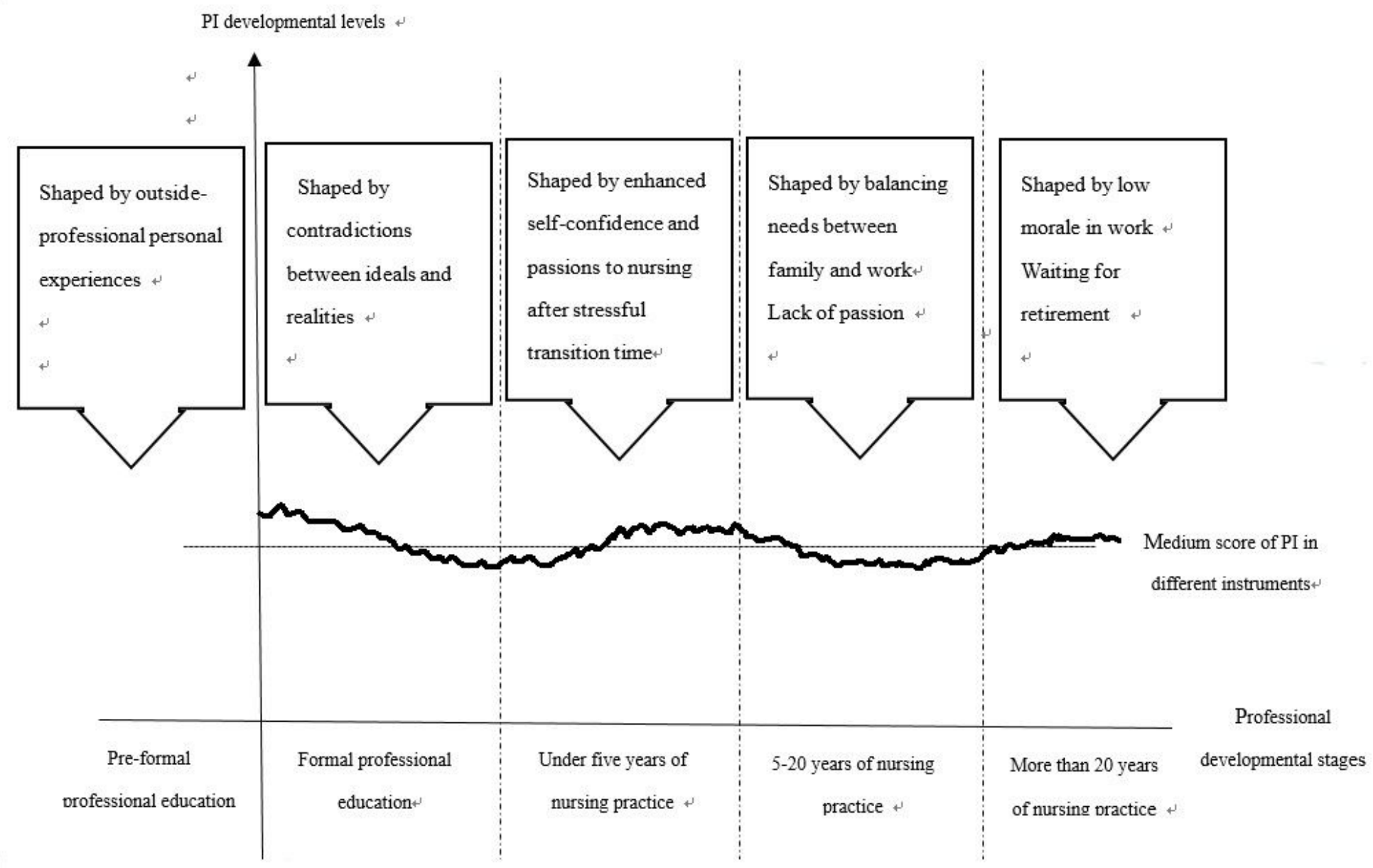




\section{Figure 3}

The PI developmental levels in different stages of career life of Chinese nurses 